\section{BASIC-PLUS programs to manage bibliographies, reference lists, and reprint files}

\author{
RICHARD DENI \\ John F. Kennedy Center for Research on \\ Education and Human Development \\ Box 154, Peabody College \\ Nashville, Tennessee 37203
}

The task of maintaining bibliographies, reference lists, and reprint files is one that few researchers and instructors enjoy. The method that is most often used is some version of an index-card system, in which individual citations, reprints, and so forth, are numbered sequentially as they are placed in the master file (Broadhurst, 1962, 1963). Each number is then added to separate subject and author files to form the basis for cross-referencing .

Bibliographies and reprint files can be maintained more completely and managed more effectively by computer, using an advanced version of BASIC. After references are coded into machine information (referred to by BASIC as "strings"), string functions can be used to sort through the reference file, selecting items based on the appearance of a combination of specific characters (search words). The full reference (author, title, journal, and key words) is subject to this kind of search.

Description. The two programs described in this paper are designed to create and manage reference files. Each file could contain references within a specific subject area. Subroutines within the programs perform the following management functions: (1) entry of individual references into a specific reference file; (2) replacement of any line of any individual reference within a file; (3) deletion of any individual reference within a file; and, (4) creation of "printfiles" from existing reference files by either "searching" the reference file for specified search words or "dumping" the entire reference file into a printfile. Whatever management option is used, the reference files and any printfiles created are always maintained in alphabetical order. Entry and management options are executed by Program 1.

The supplemental program, Program 2, allows the user to enter references from a printfile. This option bypasses individual reference entry available in Program 1.

Restrictions. These programs, written in BASIC-PLUS

Preparation of this report was supported by NICHHD Grant No. 00973.
(Digital Equipment Corporation, 1975), employ virtual core arrays as the basic structure for each reference file. Arrays are two-dimensional, with one reference per row, one line per column element. A conventional line length of 72 characters is maintained, but this figure can be extended upward. The maximum number of references per file is 140 , with a maximum of four lines for the citation and one additional line of "key words." The number of individual reference files (virtual core arrays) a user can create depends upon parameters of core storage and the availability of peripheral storage devices (mag tape, disc cartridge, etc.).

Input. The user, located at a terminal, simply types each reference along with its corresponding line of key words. Program 1 interacts with the user by asking for input and printing question marks in the left margin. The user responds by typing successive lines of the reference. We have always used the APA style citation (American Psychological Association, 1974), as it would appear on the reference page of a report. However, all letters are uppercase, and underlining is not used. Following the last line of a reference, the user enters the key word line, typing " $k w:$ : in the first three spaces of that line. Key words usually include independent or dependent variables, subject population, major theories discussed, other authors referred to in the report, and so on. Program 2 offers an additional mode of input. Program 1's "add reference" option is limited to one reference at a time. The user can save time by running Program 2, which allows creation of a printfile of references, to be entered directly from the key board. The actual entry process proceeds without the user's having to wait for each reference to be placed in the file in alphabetical order. Such a printfile can also be created as a result of the "search and print" option in Program 1. This case allows the user to move references from one file to another, or to create temporary reference files from which secondary searches can be made.

Output. Any part of a reference file or the entire file can be designated for output and printing. The "print" options of Program 1 create printfiles holding the references to be printed. The printfile may then be sent to a line printer, or listed on the user's terminal. References contained in a printfile are always maintained in alphabetical order. The user has the option of creating printfiles with or without the key word lines printed.

Other Options. Program 1 provides management options that allow the user to edit his reference files. Editing includes single-line replacement and singlereference deletion. The user finds the reference he wants to edit by searching the reference files, using the same routine used to create printfiles. 
Language and Computer. The programs are written in BASIC.PLUS and have been used extensively on a PDP 11/40 computer, under the RSTS/E operating system.

Availability. Program listings or punched tape versions are available at no charge from Richard Deni, Box 154, Peabody College, Nashville, Tennessee 37203.

\section{REFERENCES}

American Psychological Association. Publication manual (2nd ed.). Washington, D. C: Author, 1974.

Broadhurst, P. L. Coordinate indexing: A bibliographic aid. American Psychologist, 1962, 17, 137-142.

BROADHURST, P. L. Information retrieval. American Psychologist, 1963, 18, 664-665.

Digital Equipment CORPORATION. Basic-plus language manual. Maynard, Mass: July 1975. 